MSC 65K15, 65J15

\title{
THE CHEBYSHEV PROJECTIONS ON POLYHEDRON
}

\author{
V. I. ZORKALTSEV
}

Limnological Institute Siberian Branch of the Russian Academy of Sciences, Irkutsk, Russia, E-mail: vizork@mail.ru

\section{ЧЕБЫШЕВСКИЕ ПРОЕКЦИИ НА ПОЛИЭДР}

\section{В. И. ЗОРКАЛЬЦЕВ}

Федеральное государственное бюджетное учреждение науки «Лимнологический институт Сибирского отделения Российской академии наук», Иркутск, Россия, E-mail: vizork@mail.ru

Abstract. The problem of minimizing weighted Chebyshev norm on a convex polyhedron defined as the set of solutions to a system of linear inequalities may have a non-unique solution. Moreover, among the solutions to this problem, there may be clearly not suitable points of the polyhedron for the role of the closest points to the zero vector. It complicates, in particular, the Chebyshev approximation. In order to overcome the problems arising from this, the Haar condition is used, which means the requirement for the uniqueness of the solution of the indicated problem. This requirement is not always easy to verify and it is not clear what to do if it is not true. An algorithm is presented that always generates a unique solution to the indicated problem, based on the search with respect to interior points for optimal solutions of a finite sequence of linear programming problems. The solution developed is called the Chebyshev projection of the origin onto the polyhedron. It is proved that this solution is a vector of a polyhedron with Pareto-minimal absolute values of the components. It is proved that the sets of Chebyshev (according to the introduced algorithm) and Euclidean projections of the origin of coordinates onto the polyhedron, formed by varying the positive weight coefficients in the minimized Euclidean and Chebyshev norms, coincide.

KEYwords: polyhedron, the Chebyshev norms, the Chebyshev approximations.

АннотАция. Задача минимизации взвешенной чебышевской нормы на выпуклом полиэдре, определяемом как множество решений системы линейных неравенств, может иметь неединственное решение. Причем среди решений этой задачи могут оказаться явно не подходящие на роль ближайших к нулевому вектору точки полиэдра. Это затрудняет, в частности, чебышевскую 
аппроксимацию. В целях преодоления возникающих из-за этого проблем используется условие Хаара, которое означает требование единственности решения указанной задачи. Это требование не всегда легко проверить и не ясно, что делать, если оно не выполняется. Приводится алгоритм, вырабатывающий всегда единственное решение указанной задачи, основанный на поиске относительно внутренних точек оптимальных решений конечной последовательности задач линейного программирования. Вырабатываемое решение названо чебышевской проекцией начала координат на полиэдр. Доказано, что это решение является вектором полиэдра с парето-минимальными абсолютными значениями компонент. Доказано, что совпадают множества чебышевских (по введенному алгоритму) и евклидовых проекций начала координат на полиэдр, образуемые при варьировании положительных весовых коэффициентов в минимизируемых евклидовых и чебышевских нормах.

КлЮчЕВЫЕ СловА: полиэдр, чебышевские нормы, чебышевские аппроксимации.

\section{1. ВЕКТОРЫ ПОЛИЭДРА С ПАРЕТО-МИНИМАЛЬНЫМИ АБСОЛЮТНЫМИ ЗНАЧЕНИЯМИ КОМПОНЕНТ}

Рассматривается выпуклый полиэдр (далее просто полиэдр) в виде множества решений системы линейных неравенств [1]:

$$
X=\left\{x \in \mathbb{R}^{n}: A x \geq b\right\} .
$$

Заданными являются вектор $b \in \mathbb{R}^{m}$, матрица $A$ размера $m \times n$. Коэффициенты матрицы $A$ обозначим $a_{i j}, i=1, \ldots, m, j=1, \ldots, n$. Считаем, что $X \neq \varnothing$, в каждой строке матрицы $A$ есть ненулевые элементы.

Для произвольного вектора $y$ конечномерного вещественного пространства множества номеров его компонент с нулевыми,положительными, отрицательными и ненулевыми значениями обозначим

$$
\begin{array}{cc}
J_{0}(y)=\left\{j: y_{j}=0\right\}, & J_{+}(y)=\left\{j: y_{j}>0\right\}, \\
J_{-}(y)=\left\{j: y_{j}<0\right\}, & J(y)=J_{+}(y) \cup J_{-}(y) .
\end{array}
$$

Набор номеров ненулевых компонент $J(y)$ составляет [2] носитель вектоpa $y$.

Введем обозначения для множества номеров активных и неактивных неравенств для вектора $x \in X$ :

$$
I_{0}(x)=J_{0}(A x-b), \quad I(x)=J(A x-b) .
$$

Отметить, что из условия $x \in X$ следует:

$$
I(x)=J_{+}(A x-b), \quad J_{-}(A x-b)=\varnothing .
$$

Обозначим $Q$ множество парето-оптимальных решений многокритериальной задачи минимизации абсолютных значений всех компонент вектора из рассматриваемого полиэдра:

$$
\left|x_{j}\right| \rightarrow \min , \quad j \in\{1, \ldots, n\}, x \in X .
$$


Термин «парето-оптимальное решение» для данной задачи означает, что абсолютное значение любой компоненты вектора из $Q$ нельзя уменьшить, оставаясь в $X$, без увеличения абсолютного значения какой-либо другой компоненты. Иными словами, множество $Q$ состоит из векторов $x \in X$, для которых не существует вектора $q \in X$ удовлетворяющего неравенствам

$$
\sum_{j=1}^{n}\left|q_{j}\right|<\sum_{j=1}^{n}\left|x_{j}\right|,\left|q_{j}\right| \leq\left|x_{j}\right|, \quad j=1, \ldots, n .
$$

Векторы, составляющие $Q$, будем называть векторами полиэдра $X$ с парето-минимальными абсолютными значениями компонент. Из приведенного определения $Q$ непосредственно вытекает следующий критерий для идентификации векторов из $X$, не находящихся в $Q$.

Лемма 1. Вектор $x \in X$ не находится в множестве $Q$ в том и только том случае, если существует вектор $s \in \mathbb{R}^{n}, s \neq 0$ такой, что

$$
\begin{gathered}
\sum_{j=1}^{n} a_{i j} s_{j} \geq 0, \quad i \in I_{0}(x), \\
s_{j} \leq 0, \quad j \in J_{+}(x), \\
s_{j} \geq 0, \quad j \in J_{-}(x), \\
s_{j}=0, \quad j \in J_{0}(x) .
\end{gathered}
$$

Каждой системе линейных неравенств соответствует некоторая альтернативная система линейных неравенств такая, что априори известно одна и только одна из этих альтернативных систем имеет решение (см. подробнее [3]). Формулируя (по правилам из [3]) альтернативную систему линейных неравенств к системе (3)-(6), получаем следующий критерий для выявления векторов из $Q$.

Лемма 2. Вектор $x \in X$ находится в $Q$ в том и только том случае, если существует вектор $у \in \mathbb{R}^{m}$, при котором

$$
\begin{gathered}
y_{i}=0, \quad i \in I(x), \\
y_{i}>0, \quad i \in I_{0}(x), \\
c_{j}>0, \quad j \in J_{+}(x), \\
c_{j}<0, \quad j \in J_{-}(x), \\
c=A^{T} y .
\end{gathered}
$$

Свойства множества векторов полиэдра с парето-минимальными абсолютными значениями компонент исследовались в статье [4]. В частности доказано, что $Q$ замкнутое, ограниченное, связанное, возможно (обычно) не выпуклое множество, являющееся объединением конечного числа политопов, образуемых в виде выпуклых оболочек конечного числа векторов.

Задачу поиска векторов из $Q$ можно рассматривать как наиболее общую постановку проблемы определения ближайших к началу координат векторов полиэдра $X$.

Как доказано в [4] множеству $Q$ принадлежит любая евклидова проекция начала координат на полиэдр, являющаяся результатом решения задачи минимизации евклидовой нормы на полиэдре: 


$$
\left(\sum_{j=1}^{n}\left(h_{j} x_{j}\right)^{2}\right)^{1 / 2} \rightarrow \min , \quad x \in X .
$$

Здесь $h_{j}$ - заданные положительные весовые коэффициенты евклидовой нормы. Варьируя эти весовые коэффициенты, имеем разные евклидовы нормы, которые будут приводить к разным евклидовым проекциям начала координат на полиэдр. При заданном векторе весовых коэффициентов евклидова проекция существует и единственна. Такое же решение дает задача минимизации взвешенной суммы квадратов вектора из полиэдра:

$$
\sum_{j=1}^{n}\left(h_{j}\right)^{2}\left(x_{j}\right)^{2} \rightarrow \min , x \in X .
$$

Множеству $Q$ принадлежит любая октаэдральная проекция начала координат на полиэдр, т.е. любое оптимальное решение задачи минимизации октаэдральной нормы на полиэдре:

$$
\sum_{j=1}^{n} h_{j}\left|x_{j}\right| \rightarrow \min , \quad x \in X .
$$

Здесь $h_{j}$ - также заданные положительные весовые коэффициенты минимизируемой октаэдральной нормы. Данная задача всегда имеет оптимальное решение, возможно, неединственное. При любом заданном наборе положительных весовых коэффициентов $h_{j}$ все оптимальные решения данной задачи, как доказано в [5], находятся в $Q$.

Для задачи минимизации чебышевской нормы на полиэдре

$$
\max _{j=1, \ldots, n} h_{j}\left|x_{j}\right| \rightarrow \min , \quad x \in X .
$$

так же решение может быть неединственным. При этом, в отличие от задачи минимизации октаэдральной нормы, среди оптимальных решений могут оказаться векторы, не принадлежащие множеству $Q$. Такие решения явно неудовлетворительны из содержательных соображений.

Пример. Пусть $n=2$, полиэдр задан условием $x_{2} \geq 1$. При любых весовых коэфбициентах $h_{1}>0, h_{2}>0$ евклидовой проекцией начала координат на полиэдр будет вектор $\bar{x}$ с компонентами $\bar{x}_{1}=0, \bar{x}_{2}=1$. Этот же вектор будет и единственной октаэдральной проекцией начала координат на данный полиэдр при любых положительных весовых коэффициентах в октаэдральной норме. Множество векторов полиэдра с паретоминимальными абсолютными значениями компонент в этом случае состоит только из этого единственного вектора.

Задача минимизачии чебышевской нормы на таком полиэдре,

$$
\max \left\{h_{1}\left|x_{1}\right|, h_{2}\left|x_{2}\right|\right\} \rightarrow \min , x_{2} \geq 1
$$

при заданных $h_{1}>0, h_{2}>0$, даст интервал решений. Оптимальным решением будет любой вектор с компонентами $x_{2}=1, x_{1} \in\left[-h_{2} / h_{1}, h_{2} / h_{1}\right]$. $B$ данный интервал входит и вектор с компонентами $x_{2}=1, x_{1}=0$, составляющий множество $Q$. При этом входят в этот интервал и другие 
векторы, не находящиеся в $Q$, явно неподходящие для понятия «ближайшие $к$ началу координат точки полиэдра».

В целях преодоления проблем, связанных с неоднозначностью решения задачи минимизации чебышевской нормы на полиэдре, используется условие Хаара, согласно которому полиэдр должен быть таким, чтобы данная задача имела единственное решение [6-8]. Такое требование к полиэдру не всегда легко проверяемое. И при этом не ясно, что делать, если оно не выполняется.

В [9] было предложено не использовать условие Хаара, а добиваться единственного решения путем последовательного доопределения задачи поиска чебышевской проекции начала координат на полиэдр. Для приведенного примера такое доопределение состоит в минимизации на втором этапе абсолютного значения первой компоненты при зафиксированном значении по на оптимальном уровне второй компоненты. В итоге получим единственное решение $\bar{x}_{1}=0, \bar{x}_{2}=1$, которое находится в $Q$.

Для общего случая изложенный кратко в [9] алгоритм подробно представлен в следующем разделе.

\section{2. АЛГОРИТМ ВЫЧИСЛЕНИЯ ЧЕБЫШЕВСКОЙ ПРОЕКЦИИ ТОЧКИ НА пОЛИЭДР}

Алгоритм основывается на вычислении относительно внутренних точек оптимальных решений конечной последовательности задач линейного программирования с лексикографическими упорядоченными целевыми функциями. Напомним, что относительными внутренними точками называется векторы выпуклого множества, являющиеся внутренними точками этого множества относительно минимального (не сужаемого) линейного многообразия его содержащего [2].

Относительно внутренние точки множества решений системы линейных неравенств составляют решения этой системы с минимальным (не сужаемым) набором активных ограничений. Активными (выполняющимися в виде равенств) для таких решений будут только те ограничения неравенства, которые активны при любом решении рассматриваемой системы.

Отметим, что относительно внутренние точки оптимальных решений вырабатывают алгоритмы метода внутренних точек при решении задач линейного программирования.

Излагаемый ниже алгоритм был приведен в статье [10], для случая, когда вместо полиэдра рассматривается линейное многообразие, в целях иллюстрации возможных преимуществ алгоритмов внутренних точек вырабатывать именно относительно внутренние точки оптимальных решений.

Алгоритм состоит в последовательном решении задач линейного программирования с номерами $k=1, \ldots, K$, при определяемом далее числе $K$. Полагаем первоначально $k=1, J^{1}=\{j=1, \ldots, n\}, N^{1}=\varnothing$. Отметим, что рассматриваемая ниже задача линейного программирования для этапа $k=1$ является одной из равносильных формулировок задачи (12). На 
этапе $k$ решается задача:

$$
\begin{gathered}
\alpha \rightarrow \min , \\
\sum a_{i j} x_{j} \geq b_{i}, \quad i=1, \ldots, m, \\
\alpha+h_{j} x_{j} \geq 0, \quad j \in J^{k}, \\
\alpha-h_{j} x_{j} \geq 0, \quad j \in J^{k}, \\
x_{j}=\bar{x}_{j}, \quad j \in N^{k} .
\end{gathered}
$$

Искомыми являются величины $\alpha, x_{j}$ для $j \in J^{k}$. Значения $x_{j}$ для $j \in N^{k}$ зафиксированы на уровнях $\bar{x}_{j}$, определяемых ниже. Для всех этапов $k$

$$
J^{k} \cup N^{k}=\{j=1, \ldots, n\}, \quad J^{k} \cap N^{k}=\varnothing .
$$

Из (15), (16) следует, что значения целевой функции $\alpha$ задачи (13)-(17) ограничены снизу нулем. Поэтому, если имеется допустимое по условиям (14)-(17) решение, то задача (13)-(17) имеет оптимальное решение.

Для $k=1$ существование допустимого по условиям (14)-(17) решения следует из условия $X \neq \varnothing$. Для любого $x \in X$ найдется достаточно большое значение $\alpha$, при котором с данным вектором $x$ справедливы неравенства (15), (16). Условие (17) в данном случае не существенно, поскольку $N^{1}=\varnothing$. Для $k>1$ допустимое по условиям (14)-(17) решения будут составлять значения $x_{j}, j=1, \ldots, n$ и $\alpha$ полученные на предыдущем этапе вычислений.

Обозначим $\alpha^{k}, x_{j}^{k}$ для $j \in J^{k}$ оптимальное решение задачи (13)-(17) с минимальным набором активных ограничений неравенств. В качестве оптимальных будем также рассматривать значения $x_{j}^{k}$ при $j \in N^{k}$, зафиксированные по условию (17).

Множество номеров активных и неактивных ограничений неравенств (14) и (15), (16) обозначим

$$
\begin{gathered}
I_{0}^{k}=I_{0}\left(x^{k}\right), \quad I^{k}=I\left(x^{k}\right), \\
M_{-}^{k}=\left\{j \in J^{k}: h_{j} x_{j}^{k}=-\alpha^{k}\right\}, \\
M_{+}^{k}=\left\{j \in J^{k}: h_{j} x_{j}^{k}=\alpha^{k}\right\} .
\end{gathered}
$$

Одно (и возможно оба) из множеств $M_{-}^{k}, M_{+}^{k}$ не пусто. Иначе значение $\alpha^{k}$ можно было бы уменьшить, что противоречит оптимальности этого значения. Поэтому не пусто множество

$$
M^{k}=M_{-}^{k} \cup M_{+}^{k} .
$$

Положим

$$
\bar{x}_{j}=x_{j}^{k}, j \in M^{k} .
$$

Набор $M^{k}$ исключим из множества $J^{k}$ и добавим к множеству $N^{k}$. Получаем наборы номеров

$$
\begin{gathered}
J^{k+1}=J^{k} / M^{k}, \\
N^{k+1}=N^{k} \cup M^{k} .
\end{gathered}
$$


Если $J^{k+1} \neq \varnothing$, то полагаем $k:=k+1$ и переходим к следующему этапу вычислений, к поиску относительно внутренней точки оптимальных решений задачи (13)-(17) для нового значения $k$.

Поскольку $M^{k} \neq \varnothing$, то через конечное число этапов вычислений $k$ обязательно возникнет ситуация $M^{k}=J^{k}, J^{k+1}=\varnothing$. Это будет последний этап с номером $K=k$ для данного $k$.

На последнем этапе будут определены все значения $\bar{x}_{j}$ для $j=1, \ldots, n$, которые будем считать компонентами вектора $\bar{x}$ из $\mathbb{R}^{n}$. Этот вектор предлагается назвать чебышевской проекиией начала координат в $\mathbb{R}^{n}$ на полиэдр $X$.

Двойственная задача линейного программирования. В процессе решения задач линейного программирования обычно одновременно находится и решение двойственной задачи линейного программирования. Двойственной к задаче (13)-(17) будет следующая задача:

$$
\begin{gathered}
\sum_{i=1}^{m} b_{i} u_{i} \rightarrow \max , \\
\sum_{i=1}^{m} a_{i j} u_{i}+h_{j}\left(v_{j}-w_{j}\right)=0, \quad j \in J^{k}, \\
\sum_{j \in J^{k}}\left(v_{j}+w_{j}\right)=1, \\
u_{i} \geq 0 \text { для } i=1, \ldots, m, w_{j} \geq 0, v_{j} \geq 0 \text { для } j \in J^{k} .
\end{gathered}
$$

Переменными в этой задаче служат величины $u_{i}$, которые являются множителями Лагранжа условия (14) исходной задачи. Обозначим $u$ вектор в $\mathbb{R}^{m}$, составленный из этих величин. Переменными также являются величины $v_{j}$ и $w_{j}$ для $j \in J^{k}$, являющиеся множителями Лагранжа ограничений (15), (16) исходной задачи.

Будем считать, что вектор $u^{k}$ из $\mathbb{R}^{m}$ и значения $v_{j}^{k}, w_{j}^{k}$ для $j \in J^{k}$ составляют оптимальное решение двойственной задачи (25)-(28) с минимальным набором активных ограничений. Для идентификации относительно внутренних точек оптимальных решений одновременно задач (13)-(17) и (25)(28) могут служить условия дополняющей нежесткости в строгой форме. Приведем эти условия для рассматриваемых здесь задач.

Чтобы допустимые по ограничениям (14)-(17) и (26)-(28) решения исходной и двойственной задачи составляли оптимальное решение этих задач необходимо и достаточно выполнение следующих соотношений, которые составляют условия дополняющей нежесткости:

$$
\begin{gathered}
\min \left\{u_{i}^{k}, \sum_{j=1}^{n} a_{i j} x_{j}^{k}-b_{i}\right\}=0, \quad i=1, \ldots, m, \\
\min \left\{v_{j}^{k}, \alpha^{k}+h_{j} x_{j}^{k}\right\}=0, \quad j \in J^{k} \\
\min \left\{w_{j}^{k}, \alpha^{k}-h_{j} x_{j}^{k}\right\}=0, \quad j \in J^{k} .
\end{gathered}
$$


Чтобы допустимые по ограничениям (14)-(17) и (26)-(28) решения исходной и двойственной задач были оптимальными решениями этих задач с минимальными наборами активных ограничений необходимо и достаточно в дополнение к (29)-(31) выполнения неравенств:

$$
\begin{gathered}
\max \left\{u_{i}^{k}, \sum_{j=1}^{n} a_{i j} x_{j}^{k}-b_{i}\right\}>0, \quad i=1, \ldots, m, \\
\max \left\{v_{j}^{k}, \alpha^{k}+h_{j} x_{j}^{k}\right\}>0, \quad j \in J^{k}, \\
\max \left\{w_{j}^{k}, \alpha^{k}-h_{j} x_{j}^{k}\right\}>0, \quad j \in J^{k} .
\end{gathered}
$$

Неравенства (32)-(34) означают, что условия (29)-(31) выполняются в строгой форме. То есть одна и только одна из двух величин под знаком min должна быть нулевая. А вторая - обязательно положительная. Поэтому обе системы соотношений (29)-(31) и (32)-(34) называются условиями дополняющей нежесткости в строгой форме.

\section{3. ЧЕБЫШЕВСКИЕ ПРОЕКЦИИ НАЧАЛА КООРДИНАТ ЯВЛЯЮТСЯ ВЕКТОРАМИ ПОЛИЭДРА С ПАРЕТО-МИНИМАЛЬНЫМИ АБСОЛЮТНЫМИ ЗНАЧЕНИЯМИ КОМПОНЕНТ}

Изложенный в предыдущем разделе алгоритм, всегда вырабатывает единственный вектор, обозначенный $\bar{x}$. Значения компонент этого вектора зависят от изначально выбранных положительных весовых коэффициентов $h_{j}, j=1, \ldots, n$ в чебышевской норме. Справедливо следующее утверждение, объясняющее, почему вырабатываемый вектор $\bar{x}$ было предложено называть чебышевской проекцией начала координат на полиэдр.

Теорема 1. При любом наборе положительных весовых коэфбициентов $h_{j}, j=1, \ldots, n$ вырабатываемый по изложенному алгоритму вектор $\bar{x}$ будет находиться среди векторов полиэдра с парето-минимальными абсолютными значениями компонент.

Замечание 1. Отметим, что в случае, когда задача (12) имеет единственное решение, то по изложенному в предыдущем разделе алгоритму будет получено именно это решение и оно, согласно сформулированной теореме, будет находиться в $Q$.

Доказательство теоремы 1. Будем использовать решение двойственной задачи $u^{k} \in \mathbb{R}^{m}$, удовлетворяющее вместе с вектором $x^{k}$ условиям дополняющей нежесткости в строгой форме. Докажем, что существуют положительные значения $\gamma_{k}, k=1, \ldots, K$, при которых вектор

$$
y=\sum_{k=1}^{K} \gamma_{k} u^{k}
$$

будет удовлетворять (7)-(11) для $x=\bar{x}$. Это по лемме 2 означает, что $\bar{x} \in Q$.

Согласно изложенному алгоритму оптимальные значения целевой функции задачи (13)-(17) на всех этапах $k=1, \ldots, K$ неотрицательные и убывают от этапа к этапу 


$$
\alpha^{1}>\alpha^{2}>, \ldots, \alpha^{K} \geq 0
$$

Ситуачия $K=1$. Возможна ситуация, когда алгоритм завершит работу на первом этапе, т.е., когда $K=1$. Будем различать два случая.

Случай $K=1, \alpha^{k}=0$. Это означает, что $\bar{x}=0$. Такое решение будет тогда и только тогда, когда полиэдр содержит начало координат, т.е., когда $0 \in X$. А это возможно тогда и только тогда, когда у вектора $b$ нет положительных компонент, $b \leq 0$.

В этом случае условия $(7),(8)$ при $x=\bar{x}$ выполняются для $y=\gamma_{1} u^{1}$ при любом $\gamma_{1}>0$. Это следует из (28), (29), (32). Условия (9), (10) несущественны, поскольку в этом случае пусты оба множества $J_{+}(\bar{x})$ и $J_{-}(\bar{x})$.

Случай $K=1, \alpha^{k}>0$. В этом случае все компоненты вектора $\bar{x}$ ненулевые. Также для вектора $y=\gamma_{1} u^{1}$ при любом $\gamma_{1}>0$ будут выполняться условия (7), (8) при $x=\bar{x}$ согласно (28), (29), (32). Выполнение условия (9) для определяемого по правилу (11) вектора с следует из (31), (34). Выполнение условия (10) для этого вектора с следует из (30), (33).

Cuтуаиия $K>1$. Поскольку на каждом этапе $k$ определяется оптимальное решение задачи (13)-(17) с минимальным набором активных ограничений, то любое из активных ограничений неравенств (14) останется активным для любого допустимого и, следовательно, для оптимального решения задачи (13)-(17) на следующем этапе $k+1$ при $k \leq K-1$. То есть справедливы соотношения

$$
I_{0}\left(x^{k}\right) \subseteq I_{0}\left(x^{k+1}\right), \quad I\left(x^{k+1}\right) \subseteq I\left(x^{k}\right), \quad k=1, \ldots, K-1 .
$$

Следовательно

$$
I_{0}(\bar{x})=I_{0}\left(x^{K}\right), \quad I(\bar{x})=I\left(x^{K}\right) .
$$

Из (28) и соотношений $(29),(32)$ в условиях строгой дополняющей жесткости следует

$$
I_{0}\left(x^{k}\right)=J\left(u^{k}\right), \quad I\left(x^{k}\right)=J_{0}\left(u^{k}\right), \quad k=1, \ldots, K-1 .
$$

Отсюда в силу (36)

$$
J\left(u^{k}\right) \subseteq J\left(u^{k+1}\right), \quad J_{0}\left(u^{k+1}\right) \subseteq J_{0}\left(u^{k}\right), \quad k=1, \ldots, K-1 .
$$

Следовательно, для вектора $y$, определяемого по правилу (35), при любых положительных значениях $\gamma_{k}, k=1, \ldots, K$

$$
J(y)=J\left(u^{K}\right), \quad J_{0}(y)=J_{0}\left(u^{K}\right) .
$$

В силу (38)

$$
J(y)=I_{0}\left(x^{K}\right), \quad J_{0}(y)=I\left(x^{K}\right) .
$$

Из (37) получаем соотношения (7), (8) для $x=\bar{x}$ и вектора $y$, определяемого правилом (35), при любых положительных $\gamma_{k}$ :

$$
J(y)=I_{0}(\bar{x}), \quad J_{0}(y)=I(\bar{x}) .
$$


Для доказательства соотношений (9)-(11) с $x=\bar{x}$ и вектором $y$, определяемым правилом (35), при некоторых положительных $\gamma_{k}$ рассмотрим два альтернативных случая.

Случай $K>1, \alpha^{K}>0$. Рассматривается итеративный процесс формирования последовательности векторов $y^{k}$ в обратном порядке от $k=K$ до $k=1$. Пусть

$$
y^{K}=u^{K}, y^{k}=y^{k+1}+\gamma_{k} u^{k}, \quad k=K-1, \ldots, 1 .
$$

Далее определим условие, которому должен удовлетворять положительный параметр $\gamma_{k}$. Пусть

$$
s^{k}=A^{T} u^{k}, \quad k=1, \ldots, K .
$$

Процесс (39) порождает следующий процесс построения векторов $c^{k}$ от $k=K$ до $k=1$ :

$$
c^{K}=s^{K}, c^{k}=c^{k+1}+\gamma_{k} s^{k}, \quad k=K-1, \ldots, 1 .
$$

Из (26), (28), (30), (31), (33), (34) следует

$$
\begin{gathered}
s_{j}^{k}=h_{j} w_{j}^{k}>0, \quad j \in M_{+}^{k}, \quad k=1, \ldots, K, \\
s_{j}^{k}=-h_{j} v_{j}^{k}<0, \quad j \in M_{-}^{k}, k=1, \ldots, K, \\
s_{j}^{k}=0, \quad j \in J^{k+1}, \quad k=1, \ldots, K-1 .
\end{gathered}
$$

Какое бы не было значение $c_{j}^{k+1}$ при $j \in M^{k}$ существует достаточно большое значение $\gamma_{k}$ при котором

$$
\begin{gathered}
c_{j}^{k}>0, \quad j \in M_{+}^{k}, \\
c_{j}^{k}<0, \quad j \in M_{-}^{k} .
\end{gathered}
$$

И эти значение не изменяются в силу (40), (41) при переходе к предыдущим по нумерации этапам.

Так как

$$
\begin{aligned}
& J_{+}(\bar{x})=\bigcup_{k=1}^{K} M_{+}\left(x^{k}\right), \\
& J_{-}(\bar{x})=\bigcup_{k=1}^{K} M_{-}\left(x^{k}\right),
\end{aligned}
$$

то из $(42),(43)$ имеем для $c=c^{1}$

$$
\begin{aligned}
& c_{j}>0, \quad j \in J_{+}(\bar{x}), \\
& c_{j}<0, \quad j \in J_{-}(\bar{x}) .
\end{aligned}
$$

Отметим, что при $y=u^{1}$,

$$
c=A^{T} y
$$

Это означат выполнение (11). Соотношения (9), (10) для указанного выше значения $y$ и $c$ установлены.

Случай $K>1, \alpha^{K}=0$. В данном случае доказательство (9), (10) осуществляется почти по тем же правилам, что и в предыдущем. Так же по 
правилу (35) определяется вектор $y$ при $\gamma_{k}=1$ и последовательно определяются по тому же правилу величин $\gamma_{k}$ для $k=K-1, K-2, \ldots, 1$. Отличие состоит только в том, что здесь $\bar{x}_{j}=0$ для всех $j \in M^{K}$.

\section{4. СОВПАДЕНИЕ МНОЖЕСТВ ЧЕБЫШЕВСКИХ И ЕВКЛИДОВЫХ ПРОЕКЦИЙ}

В подтверждение «правомочности» используемых в данной статье правила получения всегда однозначной чебышевской проекции могут быть полезны доказываемые далее две теоремы о взаимосвязях евклидовых и чебышевских (по изложенному здесь алгоритму) проекций точки на полиэдр.

Теорема 2. Если вектор $x \in \mathbb{R}^{n}$ является евклидовой проекиией начала координат в $\mathbb{R}^{n}$ на выпуклый полиэдр $X$ при использовании в евклидовой норме вектора положительных весовых коэффициентов $h$ из $\mathbb{R}^{n}$, то этот вектор $x$ является чебышевской проекиией начала координат в $\mathbb{R}^{n}$ на полиэдр $X$ при использовании в чебышевской норме вектора положительных весовых коэфбициентов $d$ из $\mathbb{R}^{n}$ с компонентами

$$
\begin{gathered}
d_{j}=1 /\left|x_{j}\right|, \quad j \in J(x), \\
d_{j}=1, \quad j \in J_{0}(x) .
\end{gathered}
$$

Замечание 2. Случай $x=0$ не представляет интереса, поскольку тогда нулевой вектор будет евклидовой и чебышевской проекцией с любыми положительными весовыми коэффициентами в евклидовой и чебышевской нормах. В доказательстве нуждается случай $x \neq 0$. Именно он будет рассматриваться далее в доказательствах данной и последующей теорем.

Замечание 3. Согласно (44), (45) вектор $d$ зависит от значения компонент вектора $x$. По определению вектора $x$, его значения зависят от вектора весовых коэффициентов $h$. Поэтому вектор весовых коэффициентов в чебышевской норме $d$ можно считать вектор-функцией от вектора весовых коэффициентов в евклидовой норме $h$.

Доказательство теоремы 2. Поскольку вектор $x$ является точкой минимума строго выпуклой функции

$$
f(y)=\frac{1}{2} \sum_{j=1}^{n} h_{j}^{2}\left(y_{j}\right)^{2}
$$

от вектора $y$ на полиэдре $X$, то не существует направления $s \in \mathbb{R}^{n}$ такого, что

$$
\begin{gathered}
\sum a_{i j} s_{j} \leq 0, \quad i \in I_{0}(x), \\
\sum h_{j}^{2} y_{j} s_{j}<0 .
\end{gathered}
$$

Предположим, что вектор $x$ не является оптимальным решением задачи (13)-(16) с минимальным набором активных ограничений при $k=1 \mathrm{c}$ использованием весовых коэффициентов $(44),(45)$. Следовательно, существует вектор $y \in X$ такой что

$$
\frac{\left|y_{j}\right|}{\left|x_{j}\right|} \leq 1, \quad j \in J(x)
$$


и для некоторых номеров из носителя вектора $x$ это неравенство выполняется в строгой форме. Следовательно, для вектора

$$
s=y-x
$$

будут выполняться неравенства (46), (47), что противоречит определению точки $x$.

Замечание 4. Обозначим $P_{2}, P_{\infty}$ множества евклидовых и чебышевских проекций начала координат на полиэдр $X$. Эти множества образуются в результате варьирования положительных весовых коэффициентов в евклидовых и чебышевских нормах. Из теорем 1, 2 следует

$$
P_{2} \subseteq P_{\infty} \subseteq Q .
$$

Как доказано в [4], замыкание множества евклидовых проекций совпадает с подмножеством векторов полиэдра, имеющих парето-минимальные абсолютные значения компонент,

$$
\mathrm{cl} P_{2}=Q
$$

Поэтому из $(48)$ следует, что совпадают замыкания множеств $P_{2}$ и $P_{\infty}$,

$$
\operatorname{cl} P_{\infty}=Q .
$$

Согласно доказываемой ниже теореме множество $P_{\infty}$ находится в $P_{2}$ Поэтому, в уточнение (48),

$$
P_{\infty}=P_{2}
$$

Теорема 3. Если вектор $x \in \mathbb{R}^{n}$ является чебышевской проекиией начала координат в $\mathbb{R}^{n}$ на выпуклый полиэдр $X$ при исполъзовании в чебышевской норме вектора положительных весовых коэфбициентов $d$ из $\mathbb{R}^{n}$, то этот вектор $x$ является евклидовой проекцией начала координат на полиэдре $X$ при использовании в эвклидовой норме некоторого вектора положительных весовых коэфбиииентов $h$ из $\mathbb{R}^{n}$.

Доведення. На рассматриваемую задачу и алгоритм не влияет смена на противоположный знака у всех коэффициентов какого либо столбца матрицы $A$ и одновременно знака у соответствующей компоненты вектора переменных. Поэтому для упрощения записи без потери общности можем считать, что все компоненты вектора решений $x$ неотрицательные.

Итак, будем считать, что для чебышевской проекции выполняются соотношения:

$$
\begin{gathered}
\sum_{j=1}^{n} a_{i j} x_{j}=b_{j}, \quad i \in I(x), \\
\sum_{j=1}^{n} a_{i j} x_{j}<b_{j}, \quad i \in I_{0}(x), \\
d_{j} x_{j}=\alpha_{j}, \quad j \in J(x), \\
d_{j} x_{j}=0, \quad j \in J_{0}(x) .
\end{gathered}
$$


В правой части (51) находятся положительные величины $\alpha_{j}$, равные оптимальным значениям целевой функции последовательности задач (13)-(17).

Вектор $x$ является также чебышевской проекцией начала координат на полиэдр $X$ при использовании в чебышевской норме вектора весовых коэффициентов $\tilde{d}$ с компонентами

$$
\begin{gathered}
\tilde{d}_{j}=d_{j} / \alpha_{j}, \quad j \in J(x), \\
\tilde{d}_{j}=d_{j}, \quad j \in J_{0}(x) .
\end{gathered}
$$

При этом на этапе $k=1$ данный вектор $x$ будет относительно внутренней точкой оптимальных решений задач (13)-(16) с указанным вектором весовых коэффициентов. Согласно (51)-(54) выполняются соотношения

$$
\begin{gathered}
\tilde{d}_{j} x_{j}=1, \quad j \in J(x), \\
\tilde{d}_{j} x_{j}=0, \quad j \in J_{0}(x) .
\end{gathered}
$$

Оптимальное значение целевой функции имеет значение $\tilde{\alpha}=1$.

Пусть векторы $u \in \mathbb{R}^{m}, v \in \mathbb{R}^{n}, w \in \mathbb{R}^{n}$ составляют оптимальное решение двойственной к указанной выше задаче (13)-(16) этапа $k=1$ с минимальным набором активных ограничений.

Поскольку в силу (55)

$$
\tilde{\alpha}+\tilde{d}_{j} x_{j}>0, \quad \tilde{\alpha}-\tilde{d}_{j} x_{j}=0, \quad j \in J(x),
$$

то, согласно (33)

и согласно $(31),(34)$

$$
v_{j}=0, \quad j \in J(x)
$$

$$
w_{j}>0, \quad j \in J(x)
$$

Поскольку, в силу (56),

$$
\tilde{\alpha}+\tilde{d}_{j} x_{j}>0, \quad \tilde{\alpha}-\tilde{d}_{j} x_{j}>0, \quad j \in J_{0}(x),
$$

то согласно (30), (31), (33), (34)

$$
v_{j}=w_{j}=0, \quad j \in J_{0}(x) .
$$

Из (26), (57)-(59) имеем

$$
\begin{gathered}
\sum_{i=1}^{m} a_{i j} u_{i}-\tilde{d}_{j} w_{j}=0, \quad j \in J(x), \\
\sum_{i=1}^{m} a_{i j} u_{i}=0, \quad j \in J_{0}(x) .
\end{gathered}
$$

При этом согласно (29), (32)

$$
\begin{gathered}
u_{i}>0, \quad i \in I(x), \\
u_{i}=0, \quad i \in I_{0}(x) .
\end{gathered}
$$

Введем вектор положительных весовых коэффициентов $h$ с компонентами

$$
h_{j}=\left(\tilde{d}_{j} w_{j} / x_{j}\right)^{1 / 2}, \quad j \in J(x)
$$




$$
h_{j}=1, \quad j \in J_{0}(x) .
$$

Пусть

$$
f(x)=\frac{1}{2} \sum_{j=1}^{n} h_{j}^{2}\left(x_{j}\right)^{2} .
$$

Соотношения (60), (61), (64) равносильны равенству

$$
A^{T} u=\nabla f(x) .
$$

Это равенство вместе с соотношениями (44), (50), (63), (64) означают, что вектор $x$ является точкой минимума квадрата евклидовой нормы (чем является функция (66)) на полиэдре $X$.

\section{ОБСУЖДЕНИЕ}

Исследовавшийся в данной статье алгоритм можно рассматривать как обобщение рассмотренного в $[10,11]$ алгоритма определения однозначной чебышевской проекции начала координат на линейное многообразие. Доказанные в данной статье теоремы являются обобщением утверждений доказанных ранее в $[18,19]$ для проекций на линейное многообразие. Это следует из того, что выпуклый полиэдр содержит линейное многообразие как частный случай $[1,3]$.

В качестве первого по времени приложения рассматриваемой здесь задачи можно назвать проблему чебышевской аппроксимации заданной функции линейной комбинацией более удобных функций некоторого семейства.

Исторически первым [13] случаем является задача аппроксимации функции на отрезке полиномами. В дальнейшем естественным образом стали рассматриваться задачи чебышевской аппроксимации другими семействами функций, а также задачи аппроксимаций для функций от двух и большего количества переменных [7,14-19].

Для решения задач чебышевской аппроксимации используются алгоритмы, основанные на дискретизации этих задач, рассмотрения их применительно к некоторому, итеративно меняющемуся конечному набору значений аргументов. Это приводит к необходимости решения на каждой итерации рассмотренной в данной статье задачи поиска чебышевской проекции на линейное многообразие в конечномерном пространстве. При использовании и обосновании таких алгоритмов возникла проблема возможной не единственности решения у дискретизированной задачи. Это привело к введению условия Хаара [6], согласно которому дискретизированная задача всегда должна иметь единственное решение.

Для задачи аппроксимации функции одной переменой на отрезке набором из $m$ функций условие Хаара состоит в том, что любая линейная комбинация с ненулевыми всеми коэффициентами используемого набора аппроксимирующих функций должна иметь не более, чем $m-1$ нулей на 
рассматриваемом отрезке. Такие наборы функций принято называть чебышевскими системами. Это условие, как известно, выполняется при аппроксимации полиномами. Во многих случаях, например при аппроксимации сплайнами, это условие не выполняется.

В ряде работ, в том числе в статьях А. Н. Колмогорова, С. И. Зуховицкого, Р. Л. Долганова [14-16], вводились и исследовались возможности ослабления условия Хаара. Вводимые особые требования к задачам не всегда легко проверяемы и не ясно, что делать, если они не выполняются.

В данной статье, также как и в $[11,12]$ предлагается вместо введения особых требований к задачам пойти по пути развития алгоритма чебышевской аппроксимации в конечномерном случае. Из доказанных в $[11,12]$ утверждений и обобщающих их теорем, доказанных в данной статье, следует, что предложенный алгоритм дает всегда не только однозначное, но и вполне адекватное решение в конечномерном случае, вполне сопоставимое по качеству с решением которое дает, например, использование метода наименьших квадратов.

Задачи поиска чебышевской проекции точки на линейное многообразие и полиэдр в конечномерном случае возникают не только при решении проблем аппроксимации функций. Эти задачи имеют приложения в картографии (что рассматривалось еще П. Л. Чебышевым) в теории игр, в описании природных процессов, при идентификации состояний технических систем (например, электроэнергетических), при описаниях технических объектов (например, в самолетостроении).

Более подробно такие примеры приложений рассмотрены в работах ленинградской и киевской школ чебышевских аппроксимаций (см. например, $[18,19])$. В методах решения таких прикладных проблем часто используются вспомогательные задачи поиска чебышевской проекции точки на полиэдр, что обосновывает важность рассмотренного в данной статье обобщений алгоритмов из $[11,13]$.

В качестве еще одного приложения следует упомянуть использование чебышевских проекций точки на полиэдр в анализе несовместных систем линейных неравенств и несобственных задач линейного программирования в работах уральской школы исследования операций (см., например, $[1,8])$. Можно отметить, что в данном случае условие Хаара имеет вид (что отмечалось, в частности, в [20]) варианта используемого в линейном программировании условия не вырожденности.

Благодарность. Работа выполнена при финансовой поддержке фонда РФФИ № 19-07-00322 и в рамках проекта РАН № 0279-2019-0003.

\section{ЛитерАТУРА}

1. Еремин И. И. Теория линейной оптимизации. Екатеринбург: Издательство УpO PAH, 1999.

2. Рокафеллар Р. Выпуклый анализ. М.: Мир, 1973.

3. Зоркальцев В. И., Киселева М. А. Системы линейных неравенств: Учебное пособие. Иркутск: ИГУ, 2007. 
4. Зоркальцев В. И. Проекции точки на полиэдр. Журнал вычислителъной математики и математической физики. 2013. Т. 53. № 1. С. 4-19.

5. Зоркальцев В. И. Октаэдральные проекции точки на полиэдр. Журнал вычислительной математики и математической физики. 2018. Т. 58. № 5. С. $1-9$.

6. Haar A. Die Minkowskishe Geometrie und die Annäherung an stetige Funktionen. Math Ann. 1918. Vol. 78. No. 3. P. 299-311.

7. Котлатц Л., Крабе В. Теория приближений. Чебышевские приближения и их приложения. М.: Наука, 1978.

8. Черников С. Н. Линейные неравенства. М.: Наука, 1968.

9. Зоркальцев В. И. Чебышевские и другие проекции точки на полиэдр. Тезисы доклада междунар. конф. «Конструктивный негладкий анализ и смежные вопросы», посвященной памяти профессора В. Ф. Демьянова. Часть II. СПб.: Издательство ВВМ, 2017. С. 264-270.

10. Зоркальцев В. И. Метод внутренних точек: история и перспективы. Журнал вычислительной математики и математической физики. 2019. Т. 29. № 10. C. $9-25$.

11. Губий Е. В., Зоркальцев В. И., Пержабинский С. М. Чебышевские и евклидовы проекции точек на линейное многообразие. Управление большими системами. 2019. № 80. С. 6-19.

12. Зоркальцев В. И. Чебышевские проекции на линейное многообразие. Труды института математики и механики УРО РАН. 2020. № 3. С. 44-55.

13. Чебышев П. Л. Вопросы о наименьших величинах, связанные с приближенным представлением функции. Полн. собр. соч. Т. 2, М.--Л., 1947. С. 151-235.

14. Колмогоров А. Н. Замечания по поводу многочленов П. Л. Чебышева наименее уклоняющихся от заданной функции. Усn. матем. наук. 1948. T. III, вып. 1(23). C. 216-221.

15. Зуховицкий С. И., Крейн М. Г. Замечания об одном возможном обобщении теории А. Хаара и А. Н. Колмогорова. Усn. матем. наук. 1950. Т. V, вып. 1(35). C. 217-229.

16. Долганов Р. Л. Чебышевская аппроксимация асимптотически выпуклыми семействами функций. Изв. вузов. Математика. 1972. № 7. С. 35-41.

17. Александренко В. Л. Алгоритм построения приближенного равномернонаилучшего решения системы несовместных линейных уравнений. Алгоритмы и алгоритмические языки. 1968. Вып. 3. С. 57-64.

18. Демьянов В. Ф., Малоземов В. Н. Введение в минимакс. М.: Наука, 1972.

19. Каленчук-Порханова А. А. Наилучшая чебышевская аппроксимация функций одной и нескольких переменных. Кибернетика и системный анализ. 2009. № 6. C. $155-164$.

20. Левин В. Л. Применение теоремы Э. Хелли в выпуклом программировании, задачах наилучшего приближения и смежных вопросах. Математический сборник. 1969. Т. 79 (21), № 2 (6). С. 250-263.

Поступила: 01.12.2021 / Принята: 15.12.2021 


\section{ЧЕБИШОВСЬКІ ПРОЕКЦІї НА ПОЛІЕДР}

\section{В. І. ЗОРКАЛЬЦЕВ}

Федеральна державна бюджетна установа науки «Лімнологічний інститут Сибірського відділення РАН», Іркутськ, Росія, E-mail: vizork@mail.ru

АнотАція. Задача мінімізації зваженої чебишовської норми на опуклому поліедрі, який визначається як множина розв'язків системи лінійних нерівностей, може мати не єдиний розв'язок. Причому серед розв'язків цієї задачі можуть виявитись такі, що зовсім не підходять на роль найближчих до нульового вектора точок поліедра. Це ускладнює, зокрема, чебишовську апроксимацію. 3 метою подолання проблем, які при цьому виникають, використовується умова Хаара, яка означає вимогу єдиності розв'язку наведеної задачі. Цю вимогу не завжди легко перевірити і не зрозуміло що робити, якщо вона не виконується. Наведено алгоритм, який будує завжди єдиний розв'язок наведеної задачі, i заснований на пошуку відносно внутрішніх точок оптимальних розв'язків скінченої послідовності задач лінійного програмування. Розв'язок, що будується, названо чебишовською проекцією початку координат на поліедр. Доведено, що цей розв'язок є вектором поліедра з парето-мінімальними абсолютними значеннями компонент. Доведено, що співпадають множини чебишовських (за введеним алгоритмом) і евклідових проекцій початку координат на поліедр, які утворюються при варіюванні позитивних вагових коефіцієнтів в евклідових та чебишовських нормах, що мінімізуються.

КлЮчові словА: поліедр, чебишовські норми, чебишовські апроксимації. 